OPEN

SUBJECT AREAS:

SYNTHESIS OF

GRAPHENE

CANCER THERAPY

Received

30 May 2014

Accepted

2 October 2014

Published

23 October 2014

Correspondence and requests for materials should be addressed to G.G. Iggoncalves@ua. pt) or P.A.A.P.M. (paulam@ua.pt)

\section{Breakdown into nanoscale of graphene oxide: Confined hot spot atomic reduction and fragmentation}

\author{
Gil Gonçalves ${ }^{1,3}$, Mercedes Vila ${ }^{1,3}$, Igor Bdikin ${ }^{1,3}$, Alicia de Andrés ${ }^{4}$, Nazanin Emami ${ }^{5}$, \\ Rute A. S. Ferreira ${ }^{2,3}$, Luís D. Carlos ${ }^{2,3}$, José Grácio ${ }^{1,3}$ \& Paula A. A. P. Marques ${ }^{1,3}$
}

${ }^{1}$ TEMA-NRD, Mechanical Engineering Department, University of Aveiro, 3810-193 Aveiro, Portugal, ${ }^{2}$ Physics Department and CICECO, University of Aveiro, 3810-193 Aveiro, Portugal, ${ }^{3}$ Aveiro Institute of Nanotechnology, AIN, University of Aveiro, $3810-$ 193 Aveiro, Portugal, ${ }^{4}$ Instituto de Ciencia de Materiales de Madrid, Consejo Superior de Investigaciones Científicas, Cantoblanco, E-28049 Madrid, Spain, ${ }^{5}$ Department of Applied Physics and Mechanical Eng, Lulea University of Technology, Sweden.

Nano-graphene oxide (nano-GO) is a new class of carbon based materials being proposed for biomedical applications due to its small size, intrinsic optical properties, large specific surface area, and easy to functionalize. To fully exploit nano-GO properties, a reproducible method for its production is of utmost importance. Herein we report, the study of the sequential fracture of GO sheets onto nano-GO with controllable lateral width, by a simple, and reproducible method based on a mechanism that we describe as a confined hot spot atomic fragmentation/reduction of GO promoted by ultrasonication. The chemical and structural changes on GO structure during the breakage were monitored by XPS, FTIR, Raman and HRTEM. We found that GO sheets starts breaking from the defects region and in a second phase through the disruption of carbon bonds while still maintaining crystalline carbon domains. The breaking of GO is accompanied by its own reduction, essentially by the elimination of carboxylic and carbonyl functional groups. Photoluminescence and photothermal studies using this nano-GO are also presented highlighting the potential of this nanomaterial as a unique imaging/therapy platform.

$\mathrm{N}$ owadays, one of the most prompt methods to prepare graphene oxide $(\mathrm{GO})$ or reduced graphene oxide $(\mathrm{rGO})$ is through the wet chemical exfoliation of graphite. This approach has the advantage of large scale production, procedure simplicity, high efficiency and low $\operatorname{cost}^{1}$. The exfoliation of graphite in solution occurs through extensive intercalation of oxygen moieties on the aromatic structure. This increases the interplanar carbon distance and consequently weakening the interactions between adjacent carbon planes ${ }^{2}$.

The high degree of oxidation of the obtained GO grants this material with a high hydrophilic character which allows to obtain very stable aqueous colloidal suspensions ${ }^{3}$. The maximum degree of $\mathrm{GO}$ oxidation synthetized by diverse methodologies available is between $1.8<\mathrm{C} / \mathrm{O}<2.5$ ratio, usually denominated threshold oxidation degree (TOD) $)^{4}$. The surface chemistry of GO is generally described by Lerf-Klinowski model as a planar hexagonal carbon structure with hydroxyl and epoxy groups on the plane and carbonyl and carboxylic on the periphery ${ }^{5}$. Recently, Tour et al. ${ }^{6}$ suggested a dynamic structural model for GO in aqueous solution, they observed that functional groups are constantly developing and transforming by constant reactions with water. Besides, GO can be easily converted into graphene by removing a majority of the oxygen functional groups and restoring the aromatic structure through chemical ${ }^{7}$ or thermal ${ }^{8}$ reduction methods. However, the diversity of several functional groups at the GO surface could be highly desirable to build up new hybrid materials ${ }^{4,9}$. Usually two different approaches have been used for the functionalization of GO, covalent ${ }^{10-14}$ or non-covalent ${ }^{15-18}$ just depending on the final properties desired. The functionalized GO with unique and specific controlled properties has been used to build up biological platforms, biosensors and biodevices ${ }^{19-22}$.

Nano-GO, also labeled as graphene quantum dots (GQDs) can be synthetized by two different approaches: topdown or bottom-up ${ }^{23}$. Usually top-down methods consist on "cutting" graphene in very small pieces, under $100 \mathrm{~nm}$ on lateral dimensions, which could be performed by chemical ablation ${ }^{24,25}$, electrochemical oxidation ${ }^{26,27}$ or oxygen plasma treatment ${ }^{28}$. Bottom-up methods involves the synthesis of nano-GO through molecular precursors ${ }^{29-31}$.

But why "nano" Graphene Oxide? As well known, materials at nanoscale level can reveal new properties, as well as an enhancement on the properties that they already have on bulk. In case of graphene, the 2D structure of 
carbon atoms with lateral width below $100 \mathrm{~nm}$ and atomic thickness incomes high values of specific surface area and higher chemical reactivity. In fact, the morphology of nano-GO is very beneficial for interaction with biological systems ${ }^{32}$.

It was also observed that under certain critical limit size, GO can show permanent photoluminescence, arising from quantum confinement effects ${ }^{33}$. The photoluminescence of nano-GO is becoming a subject of research for bioimaging, biosensing, catalysis, photovoltaic cells and organic light emitting diodes ${ }^{23,34}$. On the other hand, GO structure also allows strong light absorption capacity in the near-infrared region (NIR) which makes it an excellent candidate for tumour cells ablation via photothermal treatment (Hyperthermia $^{35-38}$ or an effective near infrared light driven photocatalyst for selective oxidation ${ }^{39}$. Importantly, photoluminescence and NIR absorption depends on the lateral dimensions of graphene materials, but nevertheless is convenient to distinguish nano-GO materials by the high extend of lateral dimensions which enhances the appearance of these properties ${ }^{22,40}$.

Today impressive achievements have been made at the cross section between nanotechnology and biotechnology by employing carbon nanomaterials. Nano-GO is one of the most promising nanoparticles, the above referred features of nano-GO highlights its potential and points out the importance to further explore this nanomaterial in the emerging area of nanomedicine ${ }^{41}$.

This work reports a new simple, reproducible and low time consuming methodology for the synthesis of controlled size nano-GO. Chemical and structural analysis of the resulting materials allowed us to propose a mechanism to explain the GO breaking process by ultrasound in aqueous solution. The structural analysis of the final nano-GO showed that it consists on the dispersion of small domains of crystalline carbon on a matrix of amorphous carbon. The photoluminescent and NIR absorption properties that arise from this specific structure were also studied to evaluate the potential of nano-GO for bio-applications as trackable hyperthermia nanoparticles. This new material allows combining photoluminescence and NIR absorption, being from the biomaterials point of view a very interesting perspective for the development of new anticancer therapeutic concepts.

\section{Results and Discussion}

Characterization of GO breakage process. The preparation of nano-GO starts from the sequential breakage of GO sheets prepared according to a previous method reported by the authors ${ }^{42}$. By combining mechanical stirring with ultra-sonication it is possible to control the size of sheets over the time. The initial GO average size (GO1) was $\sim 390 \mathrm{~nm}$. After 30 minutes of treatment the sheets average size decreased to $\sim 200 \mathrm{~nm}(\mathrm{GO} 2)$, after 60 minutes to $\sim 140 \mathrm{~nm}$ (GO3), after 90 minutes to $\sim 60 \mathrm{~nm}$ (GO4) and finally after $180 \mathrm{~min}$ to $\sim 38 \mathrm{~nm}$ (GO5), as determined by dynamic light scattering (DLS) (Figure 1). The analysis of the inset on Figure 1 show that the size distribution of GO sheets decreases over the time of treatment, providing more accurate values at the end of the process. As it is already well known DLS analysis considers the hydrodynamic size of spherical particle models on suspension. In reality, the conformation of GO in aqueous solution depends on the chemical environment, such as ionic strength ${ }^{43}$ and $\mathrm{pH}^{44}$, varying the graphene structure from folding ${ }^{45,46}$, bending ${ }^{47}$ and scrolling ${ }^{48}$. Raymond et al. demonstrated by experimental data and molecular simulations that the planar structure of GO is less stable than bent structure $^{49}$. They consider that GO is organized in two different small domains, one's hydrophobic ( $\mathrm{sp}^{2}$ regions) and other hydrophilic ( $\mathrm{sp}^{3}$ regions, positive or negative charged, depending on $\mathrm{pH}^{50}$ ) and that this dual nature promotes the bending of GO on aqueous solution to achieve the most stable conformation state. These outcomes suggest that the present DLS measurements are valid, considering that the more stable GO structure in aqueous solution is an approximation of

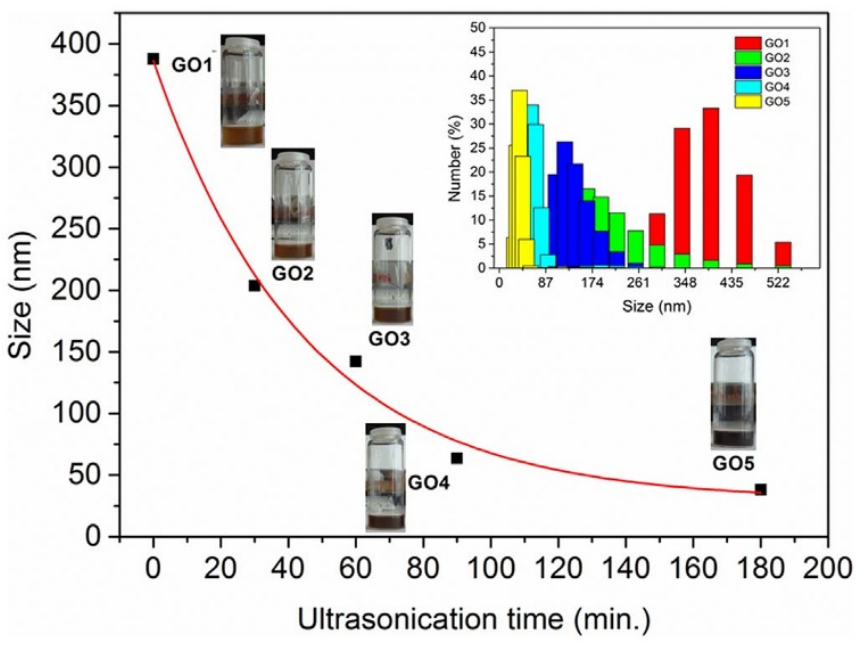

Figure $1 \mid$ Mean size of the GO sheets in function of ultra-sonication time, as obtained by DLS. The inset shows the GO size distribution for each sample, GO1, GO2, GO3, GO4 and GO5.

spherical particles instead of a completely planar structure. In the dried state, the GO sheets present very irregular 2D morphology (Figure 2, GO1) resultant from the random (irregular) chemical exfoliation of graphite. The breakage process of GO through sonication in aqueous solution showed an increase of density of small spherical particles with processing time, with more uniform particle size distribution (Figure 2, GO3). After purification of sample GO5 by centrifugation that promotes the removal of some remaining GO big flakes in solution, the average size of determined by AFM is $\sim 50 \mathrm{~nm}$ with narrow particle size distribution(Figure 2, GO5), with a process yield of around 70\%. The AFM analysis, confirms a decrease of the areas of the GO sheets during the synthesis process of nano-GO, being $2.30 \times 10^{3}$ (GO1), $0.80 \times 10^{3}$ (GO3) and $0.06 \times 10^{3} \mathrm{~nm}^{2}$ (GO5) (Figure 2).

HRTEM Images of starting material (GO1) exhibits a rough surface (Figure 3a), showing a quasi-atomic wrinkly structure at high magnification (Figure $3 \mathrm{~b}$ ). It is reported that during the drying process of GO, some solvents, in particular water, exerts surface tensions effects due to high affinity to GO oxidized domains, that can exacerbate the wrinkled structure. Beyond that, the structural defects on GO also have an important contribution on the structure deformation $^{51}$. A schematic representation of this structure is inserted (Figure 3c). In accordance to AFM analysis, the HTREM analysis of the GO5 sample shows small spherical shape nanosheets with planar structure (Figure 3d). In fact, it is not well understood why the conformation of graphene particles at nanoscale is preferentially spherical, probably some thermodynamic constrains are responsible for that behaviour. Indeed, it is well known that the spherical shape of nanoparticles allows minimal surface energy and surface stress when an interface between a solid and a fluid is considered ${ }^{52}$. High magnification images of GO5 sample shows that the disruption occurs by graphitization (Figure $3 \mathrm{~d}$ and $3 \mathrm{e}$ ). The interlayer spacing measured on the nano-GO borders is 0.34 (Figure $3 \mathrm{f}$ ), which is in accordance to the value reported for graphitic materials ${ }^{53}$.

An evaluation of the structural quality of the samples by comparing the Raman spectra of the samples after size reduction, was assessed by analysing the typical D and G bands of carbon structures (Figure S1 at supplementary information). Nano-GO Raman spectra showed the typical shape for the $488 \mathrm{~nm}$ excitation wavelength. At smaller GO sizes, there is a slight broadening of the FWHM of D peak, indicating that there is an increase of disorder or defects as expected due to the increase of GO edges with size reduction. Nevertheless, $G$ and $D$ bands do not shift as a function of the nano-GO size and $\mathrm{I}(\mathrm{D}) / \mathrm{I}(\mathrm{G})$ study as integrated areas ratio, gives 

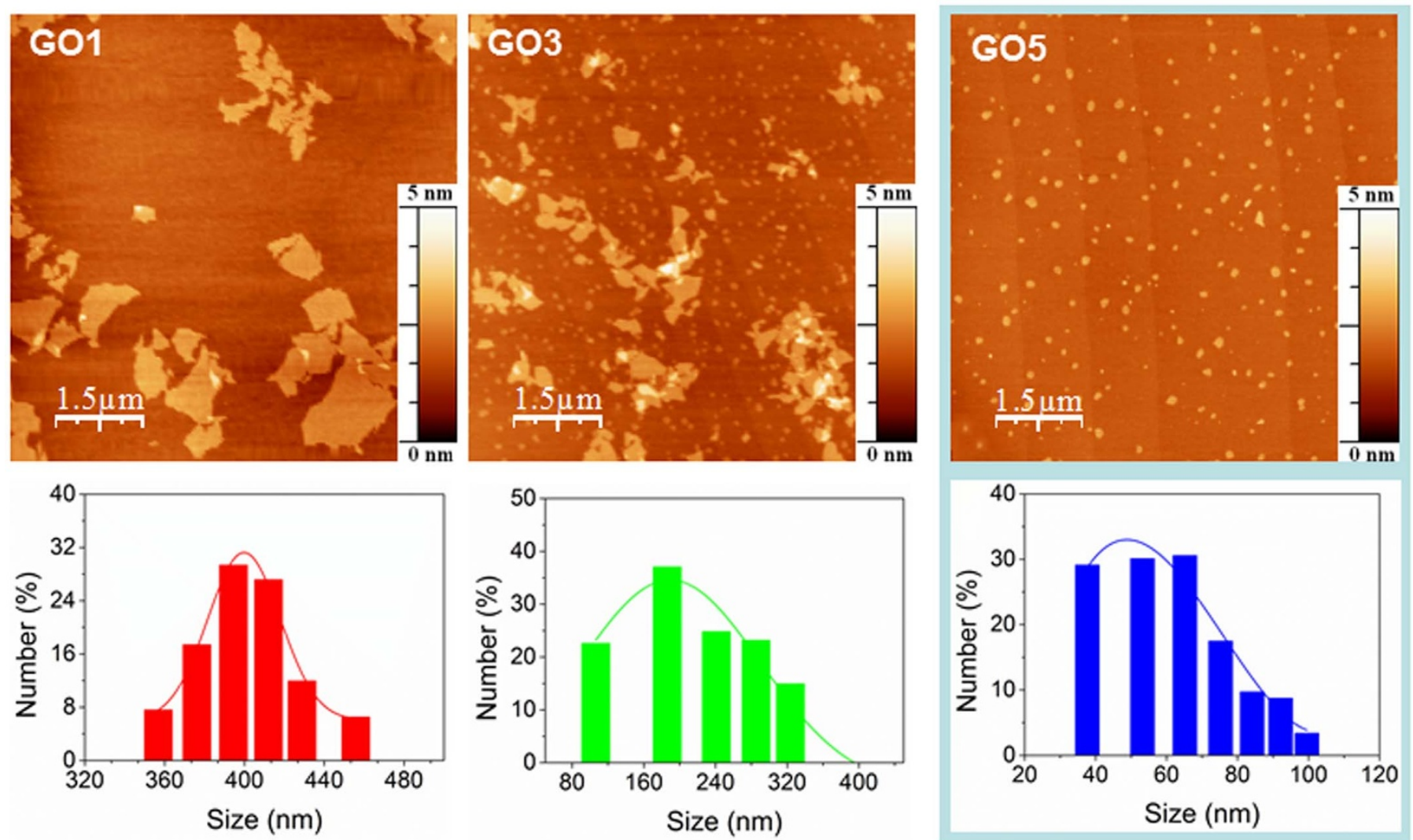

Figure 2 | Topographic view of contact-mode AFM scan of different GO samples deposited on mica substrate. The graphical representations correspond to the area distribution of the GO sheets represented for each image.

approximately stationary results for all samples suggesting that the breaking into smaller sizes does not alter the probability of finding a six fold ring on the sheets, result which is in agreement with the TEM findings.

The GO breakage occurs by applying an external source of energy that promotes the rupture of the chemical bonds, thereby facilitating its processing into nano-GO. In fact, the energy bond of the GO chemical structure depends on the oxidation state of the carbon atoms, for example $\mathrm{C}=\mathrm{C}$ bond requires $579 \mathrm{~kJ} / \mathrm{mol}$ and $\mathrm{C}-\mathrm{O}$ bond requires $383 \mathrm{~kJ} / \mathrm{mol}$ to disrupt ${ }^{54}$. The distribution of the structural defects ( $\mathrm{sp}^{3}$ carbon bonds) on the aromatic structure of GO determines therefore the appearance and propagation of cracks and consequently the final particle size ${ }^{55}$.
The GO chemical structure dynamically changes during the breaking process. An important reduction on the absorption of the -OH stretching vibrations at $3420 \mathrm{~cm}^{-1}$ and vibrations correspondent to carboxylic groups $(-\mathrm{COOH})$ at $1627 \mathrm{~cm}^{-1}$ is observed in the FTIR spectra, with the decrease of particle size (Figure 4). Interestingly, the decrease of GO particle size corresponds to an increase of the absorption bands of symmetric, asymmetric and scissoring stretching of $-\mathrm{CH}_{2}$ at 2920,2850 and $1470 \mathrm{~cm}^{-1}$. Regions of spectral overlap involving mostly $\mathrm{C}-\mathrm{O}$ and $\mathrm{C}=\mathrm{O}$ contributions $\left(850-1500 \mathrm{~cm}^{-1}\right)$ are very difficult to analyse in relation to the difference of intensities ${ }^{40}$. GO samples were further characterized by XPS, in order to obtain complementary information about the chemical structures resultant from the sequential breakage process
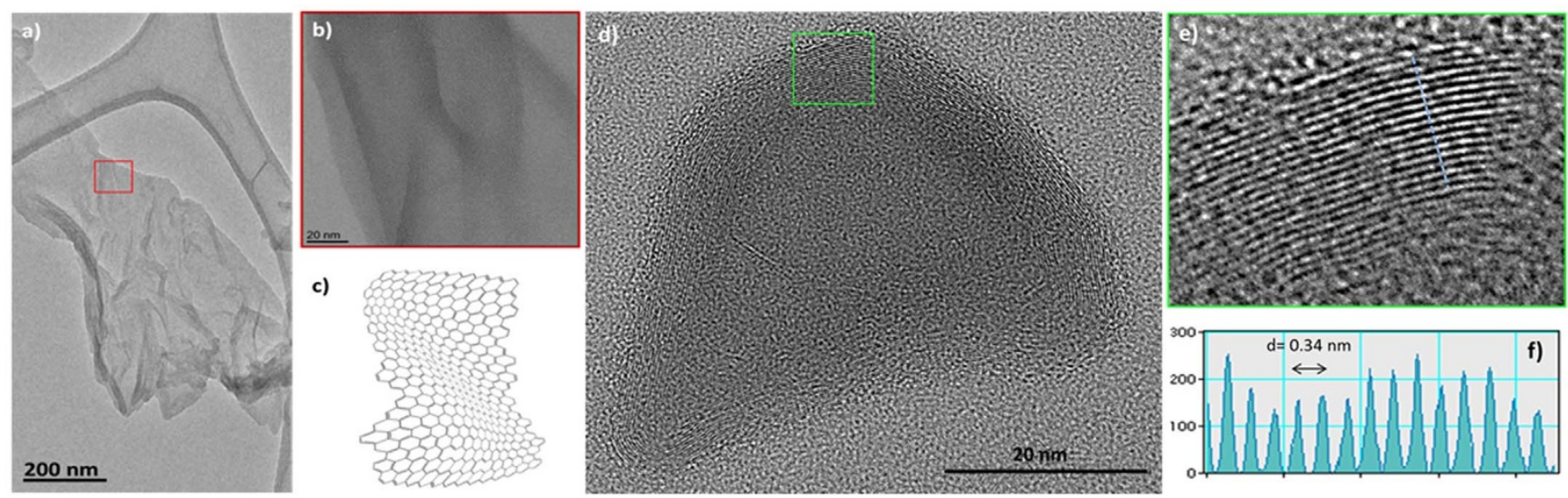

Figure 3 HRTEM images of GO1 nanosheets dispersed on the top of a carbon grid (a) and (b), with a schematic representation of the its wrinkle structure (c). HRTEM image of GO5 nanoparticle (d). High-resolution image showing the graphitic layer structure of nanoparticle (e); contrast profiles indicates the interlayer spacing $(0.34 \mathrm{~nm})$. 
(Table 1 and Figure S2). The binding energy of $\sim 285.0 \mathrm{eV}$ is attributed to the $\mathrm{C}=\mathrm{C}, \mathrm{C}-\mathrm{C}$ and $\mathrm{C}-\mathrm{H}$ bonds, the binding energies at $\sim 287.0 \mathrm{eV}$ were assigned to the $\mathrm{O}-\mathrm{C}-\mathrm{O}$ and $\mathrm{C}-\mathrm{OH}$ bonds and the binding energies at $\sim 288.7 \mathrm{eV}^{56,57}$. The samples GO1, GO2 and GO3 present a similar $\mathrm{C} / \mathrm{O}$ ratio of 1.6. However, the XPS data obtained for these 3 samples (Table 1) shows a growing conversion of $\mathrm{C}-\mathrm{O}-\mathrm{C} /$ $\mathrm{C}$ - $\mathrm{OH}$ groups onto $\mathrm{C}=\mathrm{O} / \mathrm{COOH}$ groups with treatment time. In the case of GO4 and GO5 samples the C/O ratio increases to 1.7 and 1.8 respectively, indicating a loss of oxygen containing groups and accordingly an increase of $\mathrm{C}-\mathrm{H}$ bonds (has verified by FTIR analysis) and restructuration of $\mathrm{C}-\mathrm{C}$ and $\mathrm{C}=\mathrm{C}$ bonds. Zhang et al claim that a size reduction of GO implies an increase of oxygen functional groups on its surface ${ }^{58}$, however our results for small particles (under $63.7 \mathrm{~nm}$ ) show the opposite effect.

Mechanism of GO breakage. Based on the data obtained, we postulate a possible mechanism for the breakage of GO sheets through ultrasounds. Starting from the already identified GO structure, we propose that after an initial phase of breakage, smaller sheets of GO are obtained through formation and propagation of cracks over defects zones, in particular regions with the $\mathrm{sp}^{3}$ bonds like $-\mathrm{C}-\mathrm{OH}$ and $\mathrm{O}-\mathrm{C}-\mathrm{O}$. This is schematically represented in Figure 5, where the colored lines represent the breaking areas. The final stages of breaking GO into smaller nanoparticles consist essentially in the elimination of oxygen functional groups $(\mathrm{COOH}$ and $\mathrm{C}=\mathrm{O})$ and the formation of $-\mathrm{CH}_{2}$ bonds, thereby allowing more hydrophobic materials, in accordance to XPS and FTIR results. This oxygen functional groups elimination on GO could be explained by a new mechanism that we define as a Confined Hot Spot Atomic Reduction of GO. It is well known that the use of ultrasounds promotes higher increase of local temperature and pressure that can promote reactions at atomic level, usually defined as hot spots ${ }^{59}$. On this specific case the high local energy level can promote the decomposition of water molecules onto hydroxyl radicals, those radicals have the potential to reduce carboxylic and carbonyl groups by elimination of $\mathrm{CO} / \mathrm{CO}_{2}$ and $\mathrm{H}_{2} \mathrm{O}$ and consequently allowing the restructuration of aromatic carbon structure (this effect can be observed during the reaction by the bubbles gas formation on the walls of the glass). Indeed, Acik et al. showed in his work, that the thermal reduction mechanism of GO was mainly governed thought the radical formation by the decomposition of adsorbed water on GO surface ${ }^{60}$. Those results suggest us that, instead to have a bulk source of energy that creates a homogeneous reaction medium, we have a formation of

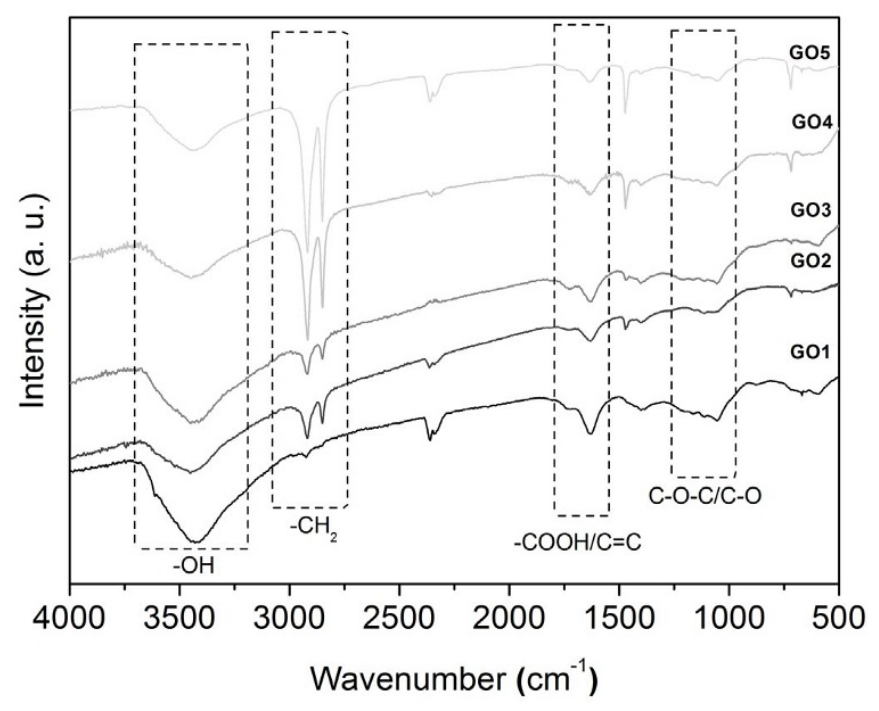

Figure $4 \mid$ FTIR spectra of the different GO samples: GO1, GO2, GO3, GO4 and GO5. instantaneous reaction spots at atomic level generated by ultrasounds that acquire the necessary thermodynamic conditions (adsorbed water + energy) for the reduction of oxygen surface groups at GO. In fact, it is also predictive that the breaking of GO in that final stage can also occurs thought the carbon double bonds. The logarithmic curve presented on Figure 1 suggests that after GO4 (size $\sim 70 \mathrm{~nm}$ ) the ultrasonication time needed to continuing breaking into small particles is much higher, which means that much more energy is needed for breaking the carbon-carbon chemical bonds $(579 \mathrm{~kJ} / \mathrm{mol} \text { for } \mathrm{C}=\mathrm{C} \text { and } 357 \mathrm{~kJ} / \mathrm{mol} \text { for } \mathrm{C}-\mathrm{C})^{54}$.

Physical properties of nano-GO. We choose GO5 sample to go further in this study and evaluate the photoluminescence and the NIR absorption properties of the prepared nano-GO. These two properties are critical for the biological applications of this nanomaterial as, for example, for photothermal therapy and imaging.

Photoluminescence of nano-GO. The optoelectronics properties of nano-GO are extremely dependent on its chemical structure (relative fraction of the $\mathrm{sp}^{2}$-hybridized domains and density of oxygen functional groups) and morphology (size, shape and number of layers $)^{51}$. In fact, the correct manipulations of GO structure during the synthesis process provide opportunities for tailoring its optoelectronic properties.

Figure 6 shows the emission spectra of GO5 (solution) under distinct excitation wavelengths. Under UV excitation (300$350 \mathrm{~nm}$ ) the spectra reveal a broad band (full-width-at-half maximum $\sim 300 \mathrm{~nm}$ ) covering the entire visible spectral range. The spectra reveal a main broad band between 500 and $800 \mathrm{~nm}$ peaking in the red spectral region at $\sim 680 \mathrm{~nm}$ and a low relative component at $\sim 440 \mathrm{~nm}$. At higher excitation wavelengths (450-475 nm) only the former component could be detected. Analogous emission components were recently reported for as-prepared GO suspended in water ${ }^{61}$. Moreover, the band within 500-800 $\mathrm{nm}$ also resembles that observed for PEGylated nano-GO ${ }^{36}$, oxygen-plasma treated ${ }^{28}$ and reduced GO being, tentatively, ascribed to the presence of induced defect-related levels in the $\pi-\pi^{*}$ bandgap $^{62}$. The low-wavelength component at $\sim 440 \mathrm{~nm}$ is usually attributed to the restructuration of $\mathrm{sp}^{3}$ onto $\mathrm{sp}^{2}$ domains on $\mathrm{GO}^{62}$. The excitation spectra were monitored within the main band (550 and $650 \mathrm{~nm}$ ) revealing a broad component (300-600 nm) with a linear plateau within $350-580 \mathrm{~nm}$, whose fwhm increases as the monitoring wavelength increases (inset in Figure 6), similarly to that previously found for GO in water ${ }^{61}$.

NIR absorption of nano-GO. The temperature variation of nanoGO aqueous solutions with distinct concentration values $(0,0.5000$, $0.0500,0.0050$ and $0.0005 \mathrm{mg} / \mathrm{mL}$ ) was measured under irradiation with a laser emitting at $980 \mathrm{~nm}$, as illustrated in the scheme in Figure S5 (supplementary information). In order to determine the solution temperature variation induced by the absorption of the NIR radiation the following preliminary experience was performed in water (in absence of GO). The temperature of the water without nano-GO $(0 \mathrm{mg} / \mathrm{mL})$ was monitored under the same experimental conditions as the ones used to monitor the temperature variation in the solutions containing different concentrations of nano-GO (Figure 7).

The influence of the nano-GO concentration on the temperature variation was evaluated by irradiating at $980 \mathrm{~nm}$ at an intensity of 1.0 $\times 10^{5} \mathrm{Wm}^{-2}$ four selected solutions with different concentrations $(0.5000,0.0500,0.0050$ and $0.0005 \mathrm{mg} / \mathrm{mL})$ during $1200 \mathrm{~s}$ (Figure 7). The temperature displays a non-linear variation, whose maximum temperature value attained depends on the nano-GO concentration. In particular, a maximum variation of the temperature of the $\mathrm{GO}$ solutions under NIR irradiation $\left(\Delta \mathrm{T}_{\mathrm{GO}}\right)$ is $283.15 \mathrm{~K}$ for $0.5000 \mathrm{mg} /$ $\mathrm{mL}$, and $280.15 \mathrm{~K}$ for $0.0500 \mathrm{mg} / \mathrm{mL}, 278.15 \mathrm{~K}$ for 0.0050 and $275.15 \mathrm{~K}$ for 0.0005 after $600 \mathrm{~s}$ of irradiation time (Figure 7 ). The 
Table 1 | Results of XPS analysis of the $\mathrm{Cl}$ s content of the surfaces of different $\mathrm{GO}$ samples

\begin{tabular}{|c|c|c|c|c|c|c|c|c|c|c|}
\hline \multirow[b]{2}{*}{ Chemical Bonds } & \multicolumn{2}{|c|}{ GO1 } & \multicolumn{2}{|c|}{ GO2 } & \multicolumn{2}{|c|}{ GO3 } & \multicolumn{2}{|c|}{ GO4 } & \multicolumn{2}{|c|}{ GO5 } \\
\hline & $\mathrm{BE}(\mathrm{eV})$ & $A C$ (at.\%) & $\mathrm{BE}(\mathrm{eV})$ & $\mathrm{AC}$ (at.\%) & $\mathrm{BE}(\mathrm{eV})$ & AC (at.\%) & $\mathrm{BE}(\mathrm{eV})$ & AC (at.\%) & $\mathrm{BE}(\mathrm{eV})$ & AC (at.\%) \\
\hline $\mathrm{C}=\mathrm{C} / \mathrm{C}-\mathrm{C} / \mathrm{C}-\mathrm{H}$ & 285.0 & 33.3 & 285.0 & 32.7 & 285.0 & 36.9 & 285.0 & 36.6 & 285.0 & 44.9 \\
\hline $\mathrm{C}=\mathrm{O} / \mathrm{COOH}$ & 288.5 & 11.9 & 288.6 & 14.4 & 288.2 & 20.8 & 288.7 & 10.4 & 288.7 & 9.7 \\
\hline $\mathrm{C} / \mathrm{O}$ ratio & \multicolumn{2}{|c|}{1.6} & \multicolumn{2}{|c|}{1.6} & \multicolumn{2}{|c|}{1.6} & \multicolumn{2}{|c|}{1.7} & \multicolumn{2}{|c|}{1.8} \\
\hline
\end{tabular}

small concentration value nano-GO $(0.0005 \mathrm{mg} / \mathrm{mL})$ should be considered a critical value for increasing the temperature of the solution.

Figure 8 shows the temperature change for the nano-GO solution with lower concentration $(0.0005 \mathrm{mg} / \mathrm{mL})$ as function of the laser irradiation time at different laser intensities. The data collected showed effectively that it is possible to achieve high temperature rates by increasing the laser power, achieving a final $\Delta \mathrm{T}_{\mathrm{GO}}$ of approximately $289.15 \mathrm{~K}$ for laser power irradiation at $2.0 \times 10^{5} \mathrm{Wm}^{-2}$.

$$
T(t)=T_{f}+\left(T_{0}-T_{f}\right) \exp \left(-\frac{h A}{c} t\right)
$$

where $\mathrm{T}_{0}$ is the temperature before irradiation $(298.15 \pm 0.1 \mathrm{~K}), \mathrm{T}_{\mathrm{f}}$ is the maximum reached temperature due to irradiation, $\mathrm{h}$ is the heat transfer coefficient in units of W. $\mathrm{K}^{-1} \cdot \mathrm{m}^{-2}$ that depends on the injected thermal power and on the thermal losses to the exterior, $\mathrm{A}$ is the area for which the heat transfers, and C is the heat capacity. Equation (1) is solution of the Newton' law of cooling and Tables S1 and S2 (Supplementary Information) list the parameters values resulting from the fit to the data in Figures 7 and 8, respectively ${ }^{63}$. We should note that from the $(\mathrm{hA}) / \mathrm{C}$ determined for water $(3.03 \times$ $10^{-3} \mathrm{~s}^{-1}$, Table $\left.\mathrm{S} 1\right)$, and considering an effective area of $\mathrm{A}=(3 \times 5)$ $\times 10-6 \mathrm{~m}^{2}$ (Figure S5), the water $\mathrm{h}$ parameter is $\sim 844 \mathrm{Wm}^{-2} \mathrm{~K}^{-1}$. This value is in good agreement with those known for water/CNTs free convective heat transfer coefficient ${ }^{64}$. Focusing our analysis on Figure 8, we note that although the maximum temperature reached increases with increasing laser power, the ratio $(\mathrm{hA}) / \mathrm{C}$ is independent of the laser power, which is expected, since those two parameters depend on material. A distinct case occurs for the data in Figure 7. In this case, the ratio (hA)/C depends on the concentration of GO. In particular, as the concentration of GO increases from 0.0005 to $0.005 \mathrm{mg} / \mathrm{mL}$ it is observed an increase of the $(\mathrm{hA}) / \mathrm{C}$, whereas at

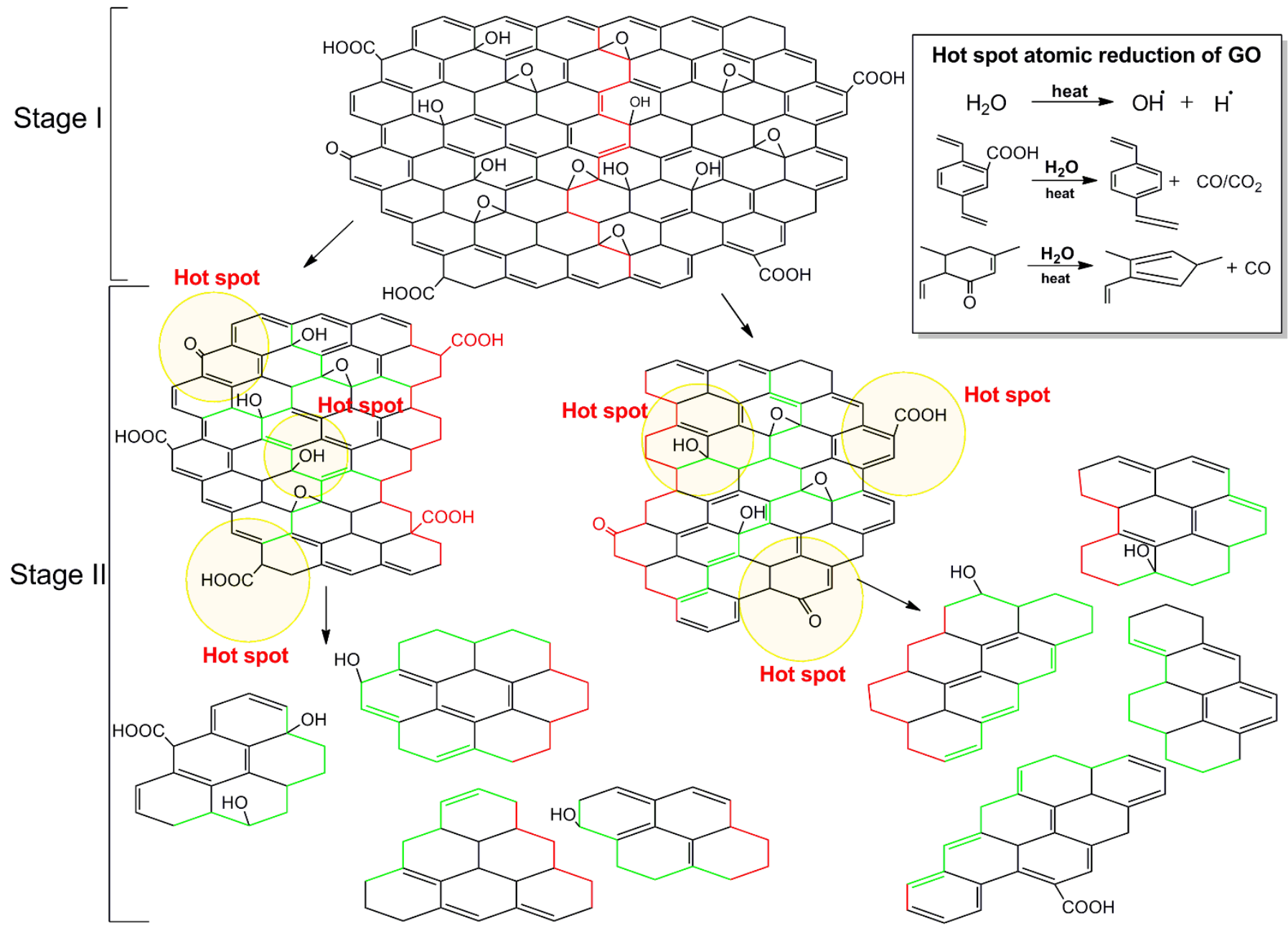

Figure $5 \mid$ Schematic representation of the breakage mechanism of GO onto nano-GO. The insect correspond to the mechanism confined hot spot atomic reduction of GO for the reduction of carboxylic and carbonyl groups. Hydroxyl and epoxy groups can probably be eliminated by this mechanism though the transformation onto carbonyl groups. Inset shows the hot spot atomic reduction reactions of GO. 


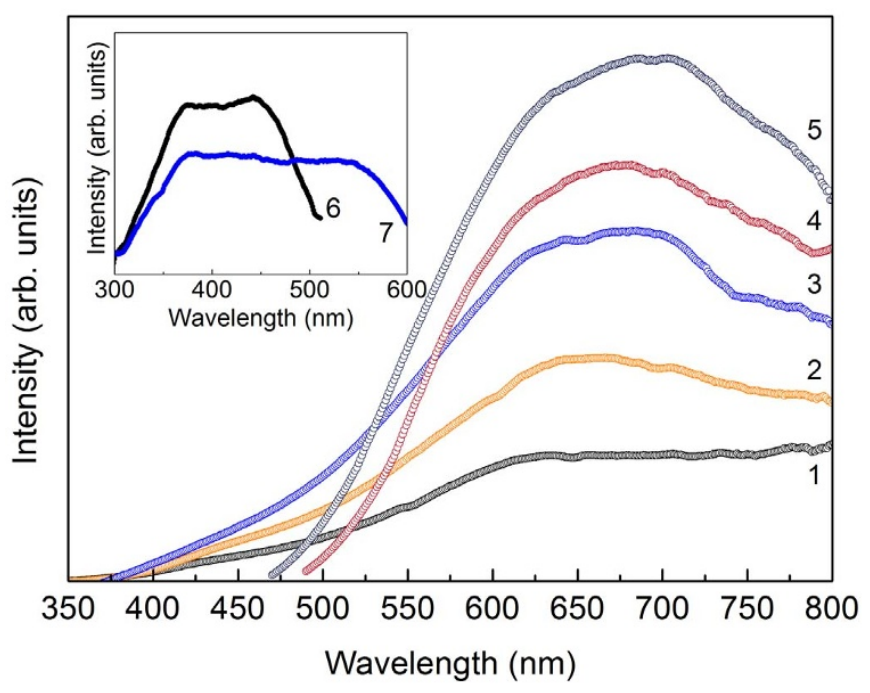

Figure 6 | Emission spectra of GO5 (solution) excited at (1) $300 \mathrm{~nm}$, (2) $325 \mathrm{~nm}$, (3) $350 \mathrm{~nm}$, (4) $450 \mathrm{~nm}$ and (5) $475 \mathrm{~nm}$. The inset shows the excitation spectra monitored at (6) $550 \mathrm{~nm}$ and (7) $650 \mathrm{~nm}$.

higher GO concentration this ratio decreases. This behavior may be rationalized as follows. For low GO concentrations, the heat transfer coefficient $\mathrm{h}$ is limited by the water thermal conductivity $\left(\mathrm{k}^{\mathrm{w}}\right)$, therefore, independent of the GO concentration. As the GO amount in solution increases from 0 to 0.0005 , the solution heat capacity decreases because $\mathrm{C}$ of $\mathrm{GO}\left(\mathrm{C}^{\mathrm{GO}}=1.7 \mathrm{JK}^{-1}\right)$ is smaller than that of the water $\left(\mathrm{C}^{\mathrm{w}}=4.18 \mathrm{JK}^{-1}\right)^{65}$. When the relative fraction of GO (f) increases (at higher GO concentrations), h will depend on the GO concentration, and the heat transfer coefficient $\mathrm{h}$ will be proportional to the thermal conductivity of the mixture $\left(\mathrm{k}^{\mathrm{mix}}\right)$, which vary within the interval: $\left(\frac{f}{K^{w}}+\frac{1-f}{K^{G O}}\right) \leq k^{m i x} \leq f k^{w}$ $+(1-f) k^{G O 66}$. Knowing that $\mathrm{k}^{\mathrm{GO}}=1.9 \mathrm{~W}^{-1} \mathrm{~K}^{-1}$ and $\mathrm{kw}=$ $0.6 \mathrm{~W}^{-1} \mathrm{~K}^{-1}$, it is expected that the $(\mathrm{hA}) / \mathrm{C}$ increases at higher GO concentrations $s^{65}$.

In summary, we propose a facile and simple approach for the synthesis of nano-GO with controlled size based on the breakage of GO sheets in aqueous solution by ultra-sonication. The analyses of the size, morphology and surface chemistry of the GO sheets in function of ultra-sonication time, suggests that the breakage of the GO sheets starts from the defect regions present on the initial structure (oxygen functional groups and structural defects). At a certain critical size limit of approximately $60 \mathrm{~nm}$, the structure starts to disrupt by the carbon bonds (double and single). The final nanoGO obtained with a mean size less than $50 \mathrm{~nm}$ is constituted by small domains of crystalline carbon dispersed on a matrix of amorphous carbon and adopt a preferential spherical shape after the breakage process. The final stages of $\mathrm{GO}$ breaking into smaller nanoparticles consist essentially in the elimination of oxygen functional groups $(\mathrm{COOH}$ and $\mathrm{C}=\mathrm{O})$ in accordance to XPS and FTIR results. These oxygen functional groups elimination from GO surface could be explained by a mechanism that we define as a confined hot spot atomic reduction of $\mathrm{GO}$ promoted by ultrasonication process.

The photoluminescence studies of the final nano-GO reveals a broad band (full-width-at-half maximum $\sim 300 \mathrm{~nm}$ ) in the visible spectral range $(500-800 \mathrm{~nm})$ with a maximum at $\sim 680 \mathrm{~nm}$ and a smaller component around $440 \mathrm{~nm}$. Besides, photothermal studies were performed by irradiating nano-GO water solutions in the NIR spectral regions. The influence of the nano-GO concentration on the solution temperature variation was evaluated through irradiation $\left(980 \mathrm{~nm}\right.$; intensity of $\left.1.0 \times 105 \mathrm{Wm}^{-2}\right)$ of four selected solutions

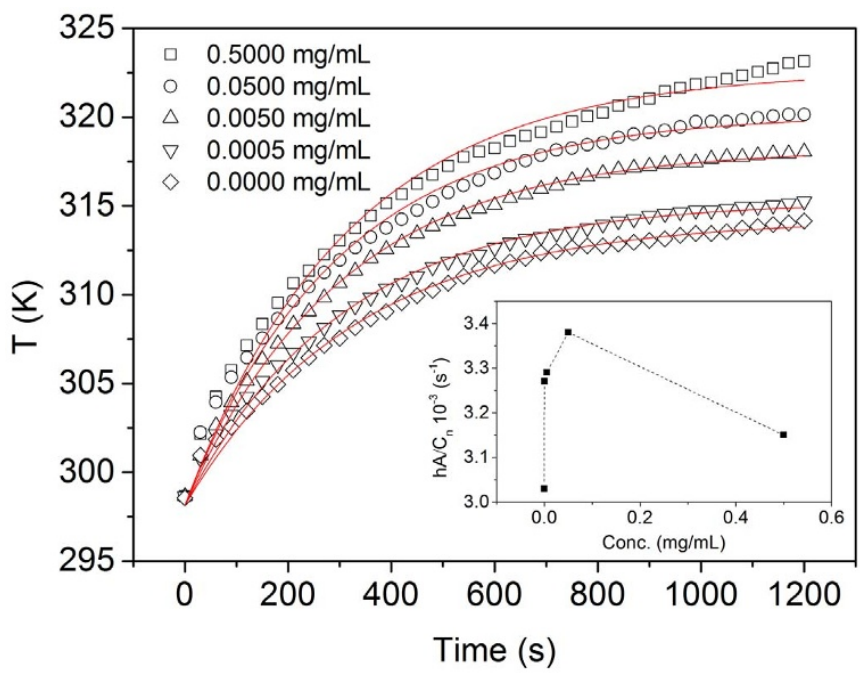

Figure $7 \mid$ Time-dependence of the temperature of the nano-GO solutions with concentration values of $0.0,0.5000,0.0500,0.0050$ and $0.0005 \mathrm{mg} / \mathrm{mL}$ under irradiation at $980 \mathrm{~nm}$ with an intensity of $1.0 \times$ $10^{5} \mathrm{Wm}^{-2}$. The solid lines correspond to the data best fit, using Equation (1). The inset shows the variation of the (hA)/C calculated from the fit. The line is a visual guide.

$(0.5000,0.0500,0.0050$ and $0.0005 \mathrm{mg} / \mathrm{mL})$. The solution temperature displays a non-linear variation depending on the nano-GO concentration. A critical value of $0.0005 \mathrm{mg} / \mathrm{mL}$ of nano-GO solution is needed to obtain a gain on energy transference from the particles to the solution. Indeed, an increase of the gain of energy transference can be achieved by increasing the laser irradiation intensity. The data collected show that it is possible to achieve high temperature rates by increasing the laser power, reaching a final $\Delta \mathrm{T}_{\mathrm{GO}}$ of approximately $16^{\circ} \mathrm{C}$ for a laser power irradiation of $2.0 \times$ $10^{5} \mathrm{Wm}^{-2}$.

Nano-GO has the central properties to be a unique imaging/therapy platform with unprecedented opportunities able for the developing advanced theranostic nano-agents.

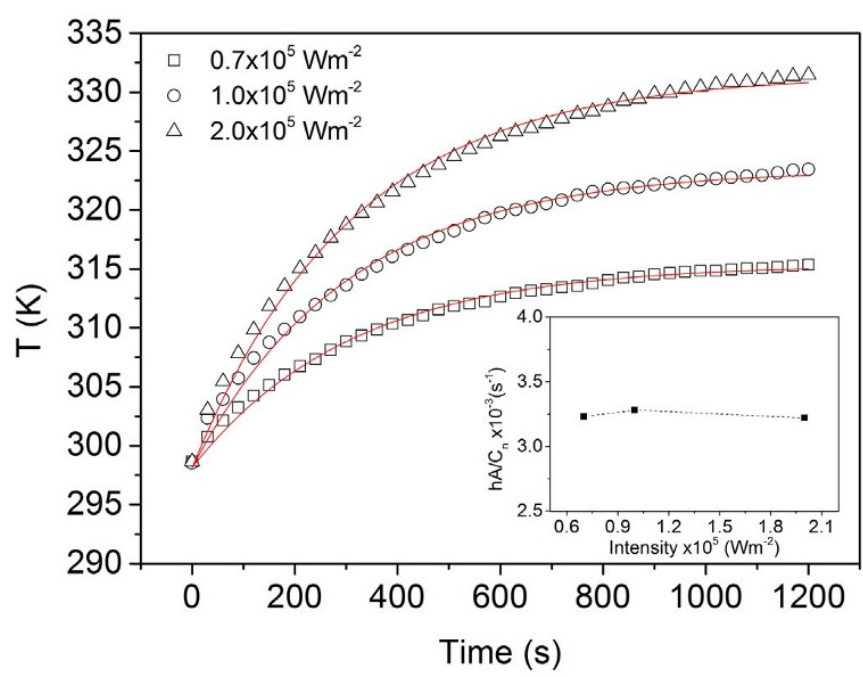

Figure $8 \mid$ Time-dependence of the temperature variation of the nanoGO solution with concentration value $0.0005 \mathrm{mg} / \mathrm{mL}$ under irradiation at $980 \mathrm{~nm}$ at distinct intensity values $\left(0.7 \times 10^{5}, 1.0 \times 10^{5}\right.$ and $2.0 \times$ $10^{5} \mathrm{Wm}^{-2}$ ). The solid lines correspond to the data best fit, using Equation (1). The inset shows the variation of the (hA)/C calculated from the fit. The line is a visual guide. 


\section{Methods}

Synthesis of nano-GO. GO was prepared by the chemical exfoliation of graphite (Graphite powder, $<45 \mu \mathrm{m},>99.99 \%$, Sigma-Aldrich) following a method described in our previous work ${ }^{42}$. Briefly, it consists on the reaction of graphite flakes with concentrated $\mathrm{H} 2 \mathrm{SO} 4$ and $\mathrm{KMnO} 4$ in order to obtain individual sheets in an oxidized state. The resultant suspension was extensively washed with distilled water by filtration and centrifugation and finally subjected to dialyses to remove ionic contaminants. The resulting GO was dried by lyophilisation to avoid aggregation. Nano-GO preparation was based on the breakage of GO by combined mechanical aging and ultra-sonication processes. $\mathrm{GO}$ was re-suspended in water and subjected to different ultra-sonication/stirring times. Five sets of different time treatments were applied, giving rise to five $\mathrm{GO}$ suspensions, herein referred to as $\mathrm{GO}$ ( 0 minutes), GO2 (30 minutes), GO3 (60 minutes), GO4 (90 minutes) and GO5 (180 minutes). The final material (GO5) was centrifuged (12000 $\mathrm{rpm} / 15 \mathrm{~min}$.) to remove possible remaining GO bigger flakes in the solution.

Nano-GO structural characterization. The surface chemistry of the different lyophilized nano-GOs was analysed by FTIR (spectra were recorded from $\mathrm{KBr}$ pellets (Aldrich, 99\%, FT-IR grade) using a Mattson 7000 FT-IR spectrometer with resolution 8 and 256 interferograms) and XPS (VG Scientific ESCALAB 200 A; UK). The size of nano-GO particles was measured by dynamic light scattering analyses (DLS) using a Zeta Sizer Nano Series (Malvern), all the samples were measured at the temperature of $25^{\circ} \mathrm{C}$ and $\mathrm{pH}$ of 6 .

Micro-Raman experiments were performed at room temperature using the $488 \mathrm{~nm}$ line of an $\mathrm{Ar}+$ laser with an incident power of $1 \mathrm{~mW}$. The light was focused and collected with an Olympus microscope ( $\times 20$ objective) and a "super-notch-plus" filter from Kaiser was used to eliminate elastic light. The scattered light was analyzed with a Jobin -Yvon HR-460 monochromator coupled to a Peltier cooled CCD.

The morphological analysis of nano-GO was performed using an atomic force microscope (AFM) (VEECO Multimode; USA) and a high resolution transmission electron microscope (HR-TEM) (JEOL 2200F; Japan) operated at $200 \mathrm{kV}$.

Nano-GO photoluminescence measurements. The photoluminescence spectra were recorded at room temperature with a modular double grating excitation spectrofluorimeter with a TRIAX 320 emission monochromator (Fluorolog-3, Horiba Scientific) coupled to a R928 Hamamatsu photomultiplier, using a front face acquisition mode. The excitation source was a $450 \mathrm{~W}$ Xe arc lamp. The emission spectra were corrected for detection and optical spectral response of the spectrofluorimeter and the excitation spectra were corrected for the spectral distribution of the lamp intensity using a photodiode reference detector.

Nano-GO photothermal measurements. The influence of the absorption in the NIR spectral region by the nano-GO on the solution temperature was performed using a laser emitting at $980 \mathrm{~nm}$ (CrystaLaser LC, DL980-3W0-TO). The laser power was quantified using a Coherent power meter (Field MaxII-TOP) coupled to a Coherent high-sensitivity silicon photodiode optical sensor (diameter of $7 \mathrm{~mm}$, OP-2 VIS). The temperature was monitored using a thermocouple thermometer (Barnant 100, model $600-2820$ ) with a resolution of $273.25 \mathrm{~K}$. Schematic representation of apparatus used to measure the temperature is shown in Figure S5 (supplementary information).

1. Park, S. \& Ruoff, R. S. Chemical methods for the production of graphenes. Nat. Nanotechnol. 4, 217-224 (2009).

2. Marcano, D. C. et al. Improved Synthesis of Graphene Oxide. ACS Nano 4, 4806-4814 (2010).

3. Paredes, J. I., Villar-Rodil, S., Martinez-Alonso, A. \& Tascon, J. M. D. Graphene oxide dispersions in organic solvents. Langmuir 24, 10560-10564 (2008).

4. Dreyer, D. R., Park, S., Bielawski, C. W. \& Ruoff, R. S. The chemistry of graphene oxide. Chem. Soc. Rev. 39, 228-240 (2010).

5. He, H. Y., Klinowski, J., Forster, M. \& Lerf, A. A new structural model for graphite oxide. Chem. Phys. Lett. 287, 53-56 (1998).

6. Dimiev, A. M., Alemany, L. B. \& Tour, J. M. Graphene Oxide. Origin of Acidity, Its Instability in Water, and a New Dynamic Structural Model. ACS Nano 7, 576-588 (2013).

7. Stankovich, S. et al. Synthesis of graphene-based nanosheets via chemical reduction of exfoliated graphite oxide. Carbon 45, 1558-1565 (2007).

8. Gomez-Navarro, C. et al. Atomic Structure of Reduced Graphene Oxide. Nano Lett. 10, 1144-1148 (2010).

9. Loh, K. P., Bao, Q., Ang, P. K. \& Yang, J. The chemistry of graphene. J. Mater. Chem. 20, 2277-2289 (2010).

10. Stankovich, S., Piner, R. D., Nguyen, S. T. \& Ruoff, R. S. Synthesis and exfoliation of isocyanate-treated graphene oxide nanoplatelets. Carbon 44, 3342-3347 (2006).

11. Niyogi, S. et al. Solution properties of graphite and graphene. J. Am. Chem. Soc. 128, 7720-7721 (2006)

12. Karousis, N., Economopoulos, S. P., Sarantopoulou, E. \& Tagmatarchis, N. Porphyrin counter anion in imidazolium-modified graphene-oxide. Carbon $\mathbf{4 8 ,}$ 854-860 (2010).

13. Zhang, X. et al. Synthesis and characterization of a graphene-C-60 hybrid material. Carbon 47, 334-337 (2009).

14. Goncalves, G. et al. Graphene oxide modified with PMMA via ATRP as reinforcement filler. J. Mater. Chem. 20, 9927-9934 (2010).
15. Patil, A. J., Vickery, J. L., Scott, T. B. \& Mann, S. Aqueous Stabilization and SelfAssembly of Graphene Sheets into Layered Bio-Nanocomposites using DNA. Adv. Mater. 21, 3159-3164 (2009).

16. Lu, C.-H., Yang, H.-H., Zhu, C.-L., Chen, X. \& Chen, G.-N. A Graphene Platform for Sensing Biomolecules. Angew. Chem.-Int. Edit. 48, 4785-4787 (2009).

17. Laaksonen, P. et al. Interfacial Engineering by Proteins: Exfoliation and Functionalization of Graphene by Hydrophobins. Angew. Chem.-Int. Edit. 49, 4946-4949 (2010).

18. Morales-Narvaez, E. \& Merkoci, A. Graphene Oxide as an Optical Biosensing Platform. Adv. Mater. 24, 3298-3308 (2012).

19. Wang, Y., Li, Z., Wang, J., Li, J. \& Lin, Y. Graphene and graphene oxide: biofunctionalization and applications in biotechnology. Trends Biotechnol. 29, 205-212 (2011).

20. Shen, H., Zhang, L., Liu, M. \& Zhang, Z. Biomedical Applications of Graphene. Theranostics 2, 283-294 (2012).

21. Zhang, Y., Nayak, T. R., Hong, H. \& Cai, W. Graphene: a versatile nanoplatform for biomedical applications. Nanoscale 4, 3833-3842 (2012).

22. Yang, K., Feng, L., Shi, X. \& Liu, Z. Nano-graphene in biomedicine: theranostic applications. Chem. Soc. Rev. 42, 530-547 (2013).

23. Shen, J., Zhu, Y., Yang, X. \& Li, C. Graphene quantum dots: emergent nanolights for bioimaging, sensors, catalysis and photovoltaic devices. Chem. Commun. 48, 3686-3699 (2012).

24. Sun, X. et al. Nano-Graphene Oxide for Cellular Imaging and Drug Delivery. Nano Res. 1, 203-212 (2008).

25. Pan, D. Y., Zhang, J. C., Li, Z. \& Wu, M. H. Hydrothermal Route for Cutting Graphene Sheets into Blue-Luminescent Graphene Quantum Dots. Adv. Mater. 22, 734-738 (2010).

26. Zhao, Q.-L. et al. Facile preparation of low cytotoxicity fluorescent carbon nanocrystals by electrooxidation of graphite. Chem. Commun. 41, 5116-5118 (2008).

27. Zheng, L. Y., Chi, Y. W., Dong, Y. Q., Lin, J. P. \& Wang, B. B. Electrochemiluminescence of Water-Soluble Carbon Nanocrystals Released Electrochemically from Graphite. J. Am. Chem. Soc. 131, 4564-4565 (2009).

28. Gokus, T. et al. Making Graphene Luminescent by Oxygen Plasma Treatment. ACS Nano 3, 3963-3968 (2009).

29. Muller, M., Kubel, C. \& Mullen, K. Giant polycyclic aromatic hydrocarbons. Chem-Euro. J. 4, 2099-2109 (1998).

30. Yang, X. et al. Two-dimensional graphene nanoribbons. J. Am. Chem. Soc. 130, 4216-4217 (2008).

31. Zhi, L. \& Muellen, K. A bottom-up approach from molecular nanographenes to unconventional carbon materials. J. Mater. Chem. 18, 1472-1484 (2008).

32. Yue, H. et al. The role of the lateral dimension of graphene oxide in the regulation of cellular responses. Biomaterials 33, 4013-4021 (2012).

33. Jin, S. H., Kim, D. H., Jun, G. H., Hong, S. H. \& Jeon, S. Tuning the Photoluminescence of Graphene Quantum Dots through the Charge Transfer Effect of Functional Groups. ACS Nano 7, 1239-1245 (2012).

34. Shen, J. H., Zhu, Y. H., Chen, C., Yang, X. L. \& Li, C. Z. Facile preparation and upconversion luminescence of graphene quantum dots. Chem. Commun. 47, 2580-2582 (2011).

35. Markovic, Z. M. et al. In vitro comparison of the photothermal anticancer activity of graphene nanoparticles and carbon nanotubes. Biomaterials 32, 1121-1129 (2011).

36. Robinson, J. T. et al. Ultrasmall Reduced Graphene Oxide with High NearInfrared Absorbance for Photothermal Therapy. J. Am. Chem. Soc. 133, 6825-6831 (2011).

37. Zhang, W. et al. Synergistic effect of chemo-photothermal therapy using PEGylated graphene oxide. Biomaterials 32, 8555-8561 (2011).

38. Yang, K. et al. The influence of surface chemistry and size of nanoscale graphene oxide on photothermal therapy of cancer using ultra-low laser power. Biomaterials 33, 2206-2214 (2012).

39. Li, H. T. et al. Near-infrared light controlled photocatalytic activity of carbon quantum dots for highly selective oxidation reaction. Nanoscale 5, 3289-3297 (2013).

40. Acik, M. et al. Unusual infrared-absorption mechanism in thermally reduced graphene oxide. Nat. Mater. 9, 840-845 (2010).

41. Gonçalves, G. et al. Nano-Graphene Oxide: A Potential Multifunctional Platform for Cancer Therapy. Adv. Healthc. Mater. 8, 1072-1090 (2013).

42. Goncalves, G. et al. Surface Modification of Graphene Nanosheets with Gold Nanoparticles: The Role of Oxygen Moieties at Graphene Surface on Gold Nucleation and Growth. Chem. Mater. 21, 4796-4802 (2009).

43. Zangmeister, C. D., Ma, X. \& Zachariah, M. R. Restructuring of Graphene Oxide Sheets into Monodisperse Nanospheres. Chem. Mater. 24, 2554-2557 (2012).

44. Whitby, R. L. D. et al. pH-driven physicochemical conformational changes of single-layer graphene oxide. Chem. Commun. 47, 9645-9647 (2011).

45. Zhang, J. et al. Free Folding of Suspended Graphene Sheets by Random Mechanical Stimulation. Phys. Rev. Lett. 104, 166805; DOI:http://dx.doi.org/10. 1103/PhysRevLett.104.166805 (2010).

46. Pandey, D. K., Chung, T. F., Prakash, G., Piner, R., Chen, Y. P. \& Reifenberger, R. Folding and cracking of graphene oxide sheets upon deposition. Surf. Sci. 605, 1669-1675 (2011).

47. Singh, M. K. et al. Atomic-scale observation of rotational misorientation in suspended few-layer graphene sheets. Nanoscale 2, 700-708 (2010). 
48. Fogler, M. M., Castro Neto, A. H. \& Guinea, F. Effect of external conditions on the structure of scrolled graphene edges. Phys. Rev. B 81, 161408; DOI:10.1103/ PhysRevB.81.161408 (2010).

49. Whitby, R. L. D. et al. Driving Forces of Conformational Changes in Single-Layer Graphene Oxide. ACS Nano 6, 3967-3973 (2012).

50. Li, D., Mueller, M. B., Gilje, S., Kaner, R. B. \& Wallace, G. G. Processable aqueous dispersions of graphene nanosheets. Nat. Nanotechnol. 3, 101-105 (2008).

51. Loh, K. P., Bao, Q., Eda, G. \& Chhowalla, M. Graphene oxide as a chemically tunable platform for optical applications. Nature Chem. 2, 1015-1024 (2010).

52. Burda, C., Chen, X. B., Narayanan, R. \& El-Sayed, M. A. Chemistry and properties of nanocrystals of different shapes. Chem. Rev. 105, 1025-1102 (2005).

53. Wen, Y., He, K., Zhu, Y., Han, F., Xu, Y., Matsuda, I., Ishii, Y., Cumings, J. \& Wang, C. Nat. Commun. 5, 1-9 (2014).

54. Batsanov, S. S. \& Batsanov, A. S. Introduction to Structural Chemistry, (Springer Dordrecht Heidelberg, 2012).

55. Pan, S. Y. \& Aksay, I. A. Factors Controlling the Size of Graphene Oxide Sheets Produced via the Graphite Oxide Route. ACS Nano 5, 4073-4083 (2011).

56. Akhavan, O. \& Ghaderi, E. Photocatalytic Reduction of Graphene Oxide Nanosheets on $\mathrm{TiO}_{2}$ Thin Film for Photoinactivation of Bacteria in Solar Light Irradiation. J. Phys. Chem. C 113, 20214-20220 (2009).

57. Yang, D. et al. Chemical analysis of graphene oxide films after heat and chemical treatments by X-ray photoelectron and Micro-Raman spectroscopy. Carbon 47, 145-152 (2009).

58. Zhang, L. et al. Size-controlled synthesis of graphene oxide sheets on a large scale using chemical exfoliation. Carbon 47, 3365-3368 (2009).

59. Xu, H. \& Suslick, K. S. Sonochemical Preparation of Functionalized Graphenes. J. Am. Chem. Soc. 133, 9148-9151 (2011).

60. Acik, M. et al. The Role of Oxygen during Thermal Reduction of Graphene Oxide Studied by Infrared Absorption Spectroscopy. J. Phys. Chem. C 115, 19761-19781 (2011).

61. Shang, J. et al. The Origin of Fluorescence from Graphene Oxide. Sci. Rep. 2, 792; DOI:10.1038/srep00792 (2012).

62. Chien, C. T. et al. Tunable Photoluminescence from Graphene Oxide. Angew. Chem.-Int. Edit. 51, 6662-6666 (2012).

63. Cengel, Y. A. \& Ghajar, A. J. Heat and Mass Transfer: Fundamentals \& Applications, [4 ${ }^{\text {th }}$ Edi.] (McGraw Hill, 2011).

64. Rashidi, F. \& Nezamabad, N. M. Experimental Investigation of Convective Heat Transfer Coefficient of CNTs Nanofluid under Constant Heat Flux Proceedings of the World Congress on Engineering 3, 1618 (2011).

65. Zhou, Y. et al. Microstructuring of Graphene Oxide Nanosheets Using Direct Laser Writing. Adv. Mater. 22, 67-71 (2010).
66. Nielsen, L. E. Predicting the properties of mixtures: mixture rules in science and engineering, [Dekker, M. (ed.)], (New York cop., 1978).

\section{Acknowledgments}

Gil Gonçalves thanks the Fundação para a Ciência e Tecnologia (FCT) for the PostDoc grant (SFRH/BDP/84419/2012). A de Andres thanks MINECO (MAT2012-37276-C03-01) project. M Vila thanks the FCT for the 2013 investigator grant. We would like to thank Fundação para a Ciência e a Tecnologia (FCT, Portugal), the European Union, QREN, FEDER, COMPETE, for funding the TEMA Research Unit (PEst-C/QUI/UI0062/2013) The authors acknowledge the SUDOE and ERDF within the Territorial Cooperation Programme IVB funding through the project CarbonInspired 2.0 (Grant Agreement SOE4/ P1/E793)

\section{Author contributions}

G.G., M.V. \& P.A.A.P.M. conceived and designed the experiments. I.B. performed the AFM experiments. A.A. did the Raman analysis. N.E. performed the XPS analysis. R.F. and L.C. made the photoluminescence and photothermal studies. J.G. involved in discussion and correction of the manuscript. All authors participated equally in discussions, analysis of the results and writing of the manuscript.

\section{Additional information}

Supplementary information accompanies this paper at http://www.nature.com/ scientificreports

Competing financial interests: The authors declare no competing financial interests. How to cite this article: Gonçalves, G. et al. Breakdown into nanoscale of graphene oxide Confined hot spot atomic reduction and fragmentation. Sci. Rep. 4, 6735; DOI:10.1038/ srep06735 (2014).

(C) $($ ) This work is licensed under a Creative Commons Attribution-NonCommercial-

BY NC No NoDerivs 4.0 International License. The images or other third party material in this article are included in the article's Creative Commons license, unless indicated otherwise in the credit line; if the material is not included under the Creative Commons license, users will need to obtain permission from the license holder in order to reproduce the material. To view a copy of this license, visit http:// creativecommons.org/licenses/by-nc-nd/4.0/ 